\title{
Cosmological Distances in Closed Model of the Universe
}

\author{
Fadel A. Bukhari \\ Department of Astronomy, Faculty of Science, King Abdulaziz University, Jeddah, Saudi Arabia \\ Email: flbukhari@hotmail.com
}

Received January 3, 2013; revised February 6, 2013; accepted February 14, 2013

Copyright (C) 2013 Fadel A. Bukhari. This is an open access article distributed under the Creative Commons Attribution License, which permits unrestricted use, distribution, and reproduction in any medium, provided the original work is properly cited.

\begin{abstract}
Four Cosmological distances were determined in the light of the closed cosmic model which was presented in a previous study. Each of these distances was obtained in terms of the redshift of an extragalactic object. It is found that the luminosity distance of the extragalactic object in the closed cosmic model, the observed model and $\Lambda=0$ model are approximately the same up to $z=0.1535$. However, the luminosity distance in the close cosmic model approximately agrees with its value in the observed model up to $z=0.6442$. Estimations of the horizon distance of the universe, the total mass and the mass of matter within the horizon distance, the equivalent numbers of the Milky Way-like galaxies and the Coma-like clusters of galaxies to the mass of matter were computed in the closed cosmic model at the present time.
\end{abstract}

Keywords: Extragalactic Distances; Cosmology; Cosmic Dynamics; Cosmological Parameter

\section{Introduction}

In [1] the proper distance, luminosity distance, angular diameter distance and distance modulus of the extragalactic object were studied in terms of its redshift in the light of five general cosmic models. In this article these cosmological distances will be investigated in the closed cosmic model which was introduced in [2]. Furthermore, interesting estimations will be determined in this model at present time. These are the horizon distance of the universe, the total mass and the mass of matter within the horizon distance, the equivalent numbers of the Milky Way-like galaxies and the Coma-like clusters of galaxies to the mass of matter. Description of methodology is illustrated in Section 2, while results and discussion are presented in Section 3, and conclusion is given in Section 4.

\section{Methodology}

The proper distance of an extragalactic object at the present time is given by

$$
d_{p}\left(t_{0}\right)=c \int_{t_{e}}^{t_{0}} \frac{\mathrm{d} t}{a(t)} .
$$

where $\mathrm{c}$ is the speed of the light and $t_{e}$ the emission time of the object photon [1]. The speed of the universe dynamics in the closed cosmic model is obtained by Equation (30) in [2] as

$$
\begin{aligned}
\dot{a}(t)= & H_{0}\left[1-s^{2} \Omega_{\Lambda, t}^{\prime}\left(1-a^{2}\right)+s^{2} \Omega_{m, t}^{\prime}\left(a^{2}-a^{3}\right)\right. \\
& \left.+s^{2} \Omega_{r, t}^{\prime}\left(a^{2}-a^{4}\right)\right]^{1 / 2} .
\end{aligned}
$$

where

$$
s=\frac{H(t)}{H_{0}} .
$$

The Hubble parameter in the expanding cosmic model at time $t$ is

$$
\begin{aligned}
H(t)= & \frac{H_{0}}{a}\left[1-\Omega_{\Lambda, o}\left(1-a^{2}\right)+\Omega_{m, o}\left(\frac{1}{a}-1\right)\right. \\
& \left.+\Omega_{r, o}\left(\frac{1}{a^{2}}-1\right)\right]^{1 / 2} .
\end{aligned}
$$

The density parameters in the expanding cosmic model at time $t$ are

$$
\begin{aligned}
& \Omega_{\Lambda, t}^{\prime}=\frac{\rho_{\Lambda, t}^{\prime}}{c^{2} \rho_{c, t}} . \\
& \Omega_{m, t}^{\prime}=\frac{\rho_{m, t}^{\prime}}{\rho_{c, t}} . \\
& \Omega_{r, t}^{\prime}=\frac{\rho_{r, t}^{\prime}}{c^{2} \rho_{c, t}} .
\end{aligned}
$$

where 


$$
\begin{array}{ccc}
\frac{\rho_{\Lambda, t}^{\prime}}{c^{2}}=\rho_{c, o} \Omega_{\Lambda, o}-\Delta\left(\frac{\rho_{\Lambda, t}}{c^{2}}\right) . & (8) & \Delta\left(\frac{\rho_{\Lambda, t}}{c^{2}}\right)=0.01 \frac{\rho_{\Lambda, t}}{c^{2}} t . \\
\rho_{m, t}^{\prime}=\rho_{c, o} \frac{\Omega_{m, o}}{a^{3}}+\frac{1}{2} \Delta\left(\frac{\rho_{\Lambda, t}}{c^{2}}\right) . & (9) & \text { where } t \text { is the cosmic time in Gyr. } \\
\frac{\rho_{r, t}^{\prime}}{c^{2}}=\rho_{c, o} \frac{\Omega_{r, o}}{a^{4}}+\frac{1}{2} \Delta\left(\frac{\rho_{\Lambda, t}}{c^{2}}\right) . & (10) \quad \text { Substituting by }(2) \text { in }(1) \text { we get } \\
d_{p}\left(t_{0}\right)=\frac{c}{H_{o}} \int_{(1+Z)^{-1}}^{1} \frac{1}{a}\left[1-s^{2} \Omega_{\Lambda, t}^{\prime}\left(1-a^{2}\right)+s^{2} \Omega_{m, t}^{\prime}\left(a^{2}-a^{3}\right)+s^{2} \Omega_{r, t}^{\prime}\left(a^{2}-a^{4}\right)\right]^{-1 / 2} \mathrm{~d} a .
\end{array}
$$

The density parameters in the closed cosmic model at time $t$ are

$$
\begin{aligned}
& \Omega_{\Lambda, t}^{\prime \prime}=\frac{\rho_{\Lambda, t}^{\prime}}{c^{2} \rho_{c, t}^{\prime \prime}} . \\
& \Omega_{m, t}^{\prime \prime}=\frac{\rho_{m, t}^{\prime}}{\rho_{c, t}^{\prime \prime}} .
\end{aligned}
$$

where

$$
\Omega_{r, t}^{\prime \prime}=\frac{\rho_{r, t}^{\prime}}{c^{2} \rho_{c, t}^{\prime \prime}}
$$

$$
\rho_{c, t}^{\prime \prime}=\frac{3 H^{\prime 2}(t)}{8 \pi G} .
$$

and the Hubble parameter in the closed cosmic model at time $t$ is

$$
H^{\prime}(t)=\frac{H_{o}}{a}\left[1-s^{2} \Omega_{\Lambda, t}^{\prime}\left(1-a^{2}\right)+s^{2} \Omega_{m, t}^{\prime}\left(a^{2}-a^{3}\right)+s^{2} \Omega_{r, t}^{\prime}\left(a^{2}-a^{4}\right)\right]^{1 / 2} .
$$

using (5), (14) we have

$$
\frac{\Omega_{\Lambda, t}^{\prime \prime}}{\Omega_{\Lambda, t}^{\prime}}=\frac{\rho_{c, t}}{\rho_{c, t}^{\prime \prime}} .
$$

substituting by (12), (17) in (19) we get

Using (3)

$$
\begin{gathered}
\frac{\Omega_{\Lambda, t}^{\prime \prime}}{\Omega_{\Lambda, t}^{\prime}}=\frac{H^{2}}{H^{\prime 2}} \\
\Omega_{\Lambda, t}^{\prime} H^{2} s^{2}=\Omega_{\Lambda, t}^{\prime \prime} H^{\prime 2} s^{2}
\end{gathered}
$$

$$
s^{2} \Omega_{\Lambda, t}^{\prime}=\frac{H^{\prime 2}}{H_{o}^{2}} \Omega_{\Lambda, t}^{\prime \prime}
$$$$
d_{p}\left(t_{o}\right)=\frac{c}{H_{o}} \int_{(1+z)^{-1}}^{1} \frac{1}{a}\left[1-S^{2} \Omega_{\Lambda, t}^{\prime \prime}\left(1-a^{2}\right)+S^{2} \Omega_{m, t}^{\prime \prime}\left(a^{2}-a^{3}\right)+S^{2} \Omega_{r, t}^{\prime \prime}\left(a^{2}-a^{4}\right)\right]^{-1 / 2} \mathrm{~d} a .
$$

Let $S=\frac{H^{\prime}}{H_{o}}$, then Equation (20) can be written as

$$
S^{2} \Omega_{\Lambda, t}^{\prime}=S^{2} \Omega_{\Lambda, t}^{\prime \prime}
$$

Similarly we can get

$$
\begin{gathered}
s^{2} \Omega_{m, t}^{\prime}=S^{2} \Omega_{m, t}^{\prime \prime} . \\
s^{2} \Omega_{r, t}^{\prime}=S^{2} \Omega_{r, t}^{\prime \prime} .
\end{gathered}
$$

Substituting by (21)-(23) in (13) the proper distance of the extragalactic abject in the closed cosmic model at the present time becomes

and its luminosity distance, angular diameter distance and distance modulus are respectively

$$
\begin{aligned}
& d_{L}\left(t_{o}\right)=\frac{c(1+z)}{H_{o}} \int_{(1+z)^{-1}}^{1} \frac{1}{a}\left[1-S^{2} \Omega_{\Lambda, t}^{\prime \prime}\left(1-a^{2}\right)+S^{2} \Omega_{m, t}^{\prime \prime}\left(a^{2}-a^{3}\right)+S^{2} \Omega_{r, t}^{\prime \prime}\left(a^{2}-a^{4}\right)\right]^{-1 / 2} \mathrm{~d} a . \\
& d_{A}\left(t_{o}\right)=\frac{c}{H_{o}(1+z)} \int_{(1+z)^{-1}}^{1} \frac{1}{a}\left[1-S^{2} \Omega_{\Lambda, t}^{\prime \prime}\left(1-a^{2}\right)+S^{2} \Omega_{m, t}^{\prime \prime}\left(a^{2}-a^{3}\right)+S^{2} \Omega_{r, t}^{\prime \prime}\left(a^{2}-a^{4}\right)\right]^{-1 / 2} \mathrm{~d} a . \\
& m-M=25+5 \log \left[\frac{c(1+z)}{H_{o}} \int_{(1+z)^{-1}}^{1} \frac{1}{a}\left\{1-S^{2} \Omega_{\Lambda, t}^{\prime \prime}\left(1-a^{2}\right)+S^{2} \Omega_{m, t}^{\prime \prime}\left(a^{2}-a^{3}\right)+S^{2} \Omega_{r, t}^{\prime \prime}\left(a^{2}-a^{4}\right)\right\}^{-1 / 2} \mathrm{~d} a\right] .
\end{aligned}
$$


The horizon distance of the universe in the closed cosmic model at the present time is given by Equation (28).

It is obvious from Table (3) in [2] that the space of the universe is flat at the present time. Hence, as explained in details in [1] the volume of sphere of radius $d_{h}\left(t_{o}\right)$ is expressed as

$$
V_{h}\left(t_{o}\right)=\frac{8 \pi}{3} d_{h}^{3}\left(t_{o}\right)
$$

The total density of the universe in the closed cosmic model at time $t$ is

$$
\rho^{\prime}(t)=\rho_{m, t}^{\prime}+\frac{\rho_{r, t}^{\prime}}{c^{2}}+\frac{\rho_{\Lambda, t}^{\prime}}{c^{2}}
$$

Substituting by (14)-(16) in (30) we get

$$
\begin{gathered}
\rho^{\prime}(t)=\rho_{c, t}^{\prime \prime} \Omega_{m, t}^{\prime \prime}+\rho_{c, t}^{\prime \prime} \Omega_{r, t}^{\prime \prime}+\rho_{c, t}^{\prime \prime} \Omega_{\Lambda, t}^{\prime \prime} \\
\rho^{\prime}(t)=\rho_{c, t}^{\prime \prime} \Omega^{\prime \prime}(t) .
\end{gathered}
$$

where $\Omega^{\prime \prime}(t)=\Omega_{m, t}^{\prime \prime}+\Omega_{r, t}^{\prime \prime}+\Omega_{\Lambda, t}^{\prime \prime}$. At the present time we have

$$
\rho^{\prime}\left(t_{o}\right)=\rho_{c, t_{o}}^{\prime \prime} \Omega^{\prime \prime}\left(t_{o}\right) .
$$

Hence, the total mass and the mass of matter within $d_{h}\left(t_{0}\right)$ in the closed cosmic model at the present time are respectively expressed as

$$
\begin{gathered}
M_{h}\left(t_{o}\right)=V_{h}\left(t_{o}\right) \rho^{\prime}\left(t_{o}\right) . \\
M_{m}\left(t_{o}\right)=M_{h}\left(t_{o}\right) \frac{\Omega_{m, t_{o}}^{\prime \prime}}{\Omega^{\prime \prime}\left(t_{o}\right)} .
\end{gathered}
$$

The equivalent numbers of the Milky Way-like galaxies and the Coma-like clusters to $M_{m}\left(t_{o}\right)$ are respectively written as

$$
\begin{aligned}
& N_{M W}=\frac{M_{m}\left(t_{o}\right)}{M_{M W}} . \\
& N_{\text {COMA }}=\frac{M_{m}\left(t_{o}\right)}{M_{\text {COMA }}} .
\end{aligned}
$$

where $M_{M W}, M_{\text {COMA }}$ are the masses of the Milky Way galaxy and the Coma cluster of galaxies respectively.

\section{Results and Discussion}

Results of the closed cosmic model would be compared with those of the general cosmic model A which best represents observations $[2,3]$.

The proper distance-redshift relation (24) in the closed cosmic model and the corresponding relation in the general cosmic model $A$ [1] up to $z=6$ are plotted in
Figure 1. It is found that both distributions increase with $z$ and they coincide on each other for $z \leq 1.0098$, but they gradually diverge from each other afterwards, such that the distribution of the general model A becomes slightly upper the distribution of the closed model. This indicates that for $z>1.0098$ the proper distance of extragalactic object in the closed cosmic model is slightly less than its value in the general cosmic model $\mathrm{A}$, that is because the effect of slow transfer of dark energy density into matter and radiation densities which becomes prominent for $z>$ 1.0098 .

The luminosity distance-redshift relation (25) in the closed cosmic model and the corresponding relations in the general cosmic model $\mathrm{A}$ and the $\Lambda=0$ model [1] up to $z=1$ are represented in Figure 2. The three distributions increase with $z$ and coincide on each other up to $z=0.1535$. However, the distribution of $\Lambda=0$ model starts diverging from the distributions of the first two models for $z>0.1535$, such that it becomes lower them. Furthermore, at $z=0.6442$ the distribution of the closed model gradually diverges form that of the general model A and becomes slightly lower it. This is also because the effect of minor transfer of the dark energy density into matter and radiation densities which gets noticeable for $z$ $>0.6442$.

The angular diameter distance-redshift relation (26) in the closed cosmic model and the corresponding relation in the general cosmic model and A [1] up to $z=6$ are illustrated in Figure 3. Both distributions display steep increase with $z$ up to $z=0.9857$ then the closed model distribution reaches its maximum $\left(d_{A_{\max }}=1727.3954 \mathrm{Mpc}\right)$ at $z=1.6083$, while the general model $\mathrm{A}$ distribution gets its maximum

$\left(d_{A_{\max }}=1765.5434 \mathrm{Mpc}\right)$ at $Z=1.6442$ as given in [1]. The distributions coincide on each other up to $Z=0.6857$. However, the distribution of the closed model gradually deflects from that of the general model A for $z \geq 0.6857$ and remains lower it. Again the only reason for this result is the slight transfer of dark energy density into matter and radiation densities which becomes obvious for $z>$ 0.6857 .

The distance modulus-redshift relation (27) in the closed cosmic model and the corresponding relation in the general cosmic model A [1] up to $z=1$ are shown in Figure 4. The distributions coincide on each other without any significant diversion, as slight differences in the luminosity distances of the two models at given value of $z$ will disappear in the logarithms of the distance modulus relations. Furthermore, in the closed cosmic model relations (28), (33)-(36) give the following cosmological estimations respectively at the present time:

$$
d_{h}\left(t_{o}\right)=\frac{c}{H_{o}} \int_{0}^{1} \frac{1}{a}\left[1-S^{2} \Omega_{\Lambda, t}^{\prime \prime}\left(1-a^{2}\right)+S^{2} \Omega_{m, t}^{\prime \prime}\left(a^{2}-a^{3}\right)+S^{2} \Omega_{r, t}^{\prime \prime}\left(a^{2}-a^{4}\right)\right]^{-1 / 2} \mathrm{~d} a .
$$




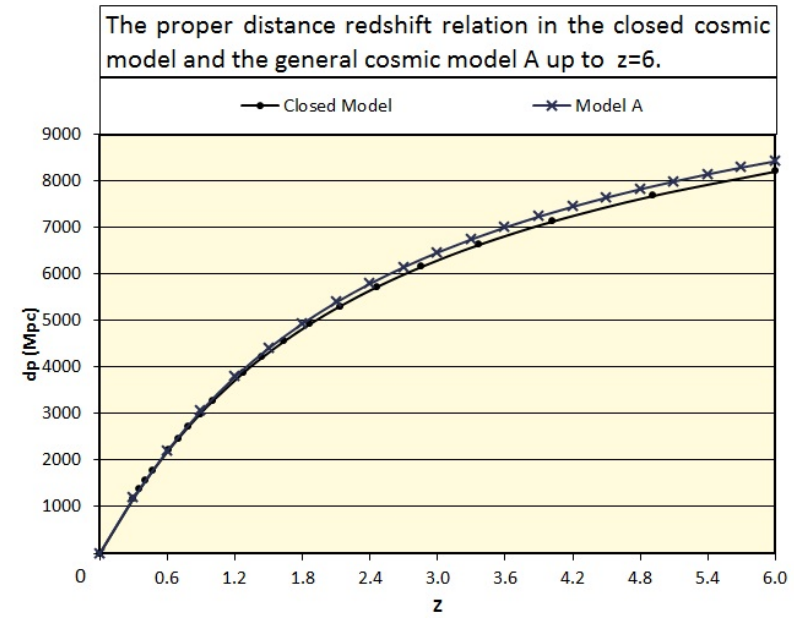

Figure 1. The proper distance redshift relation in the closed cosmic model and the general cosmic model $A$ up to $z=6$.

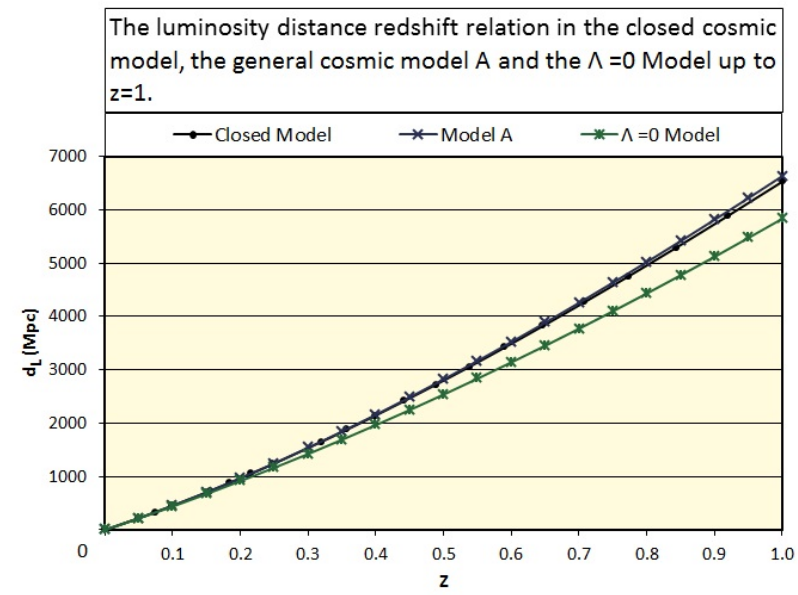

Figure 2. The luminosity distance redshift relation in the closed cosmic model, the general cosmic model $A$ and the $\Lambda$ $=0$ model up to $\mathrm{z}=1$.

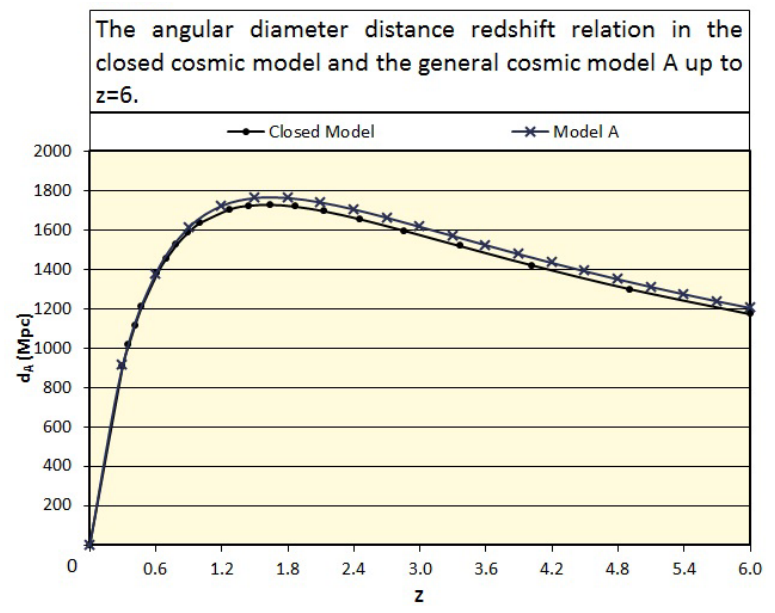

Figure 3. The angular diameter distance redshift relation in the closed cosmic model and the general cosmic model $A$ up to $z=6$.

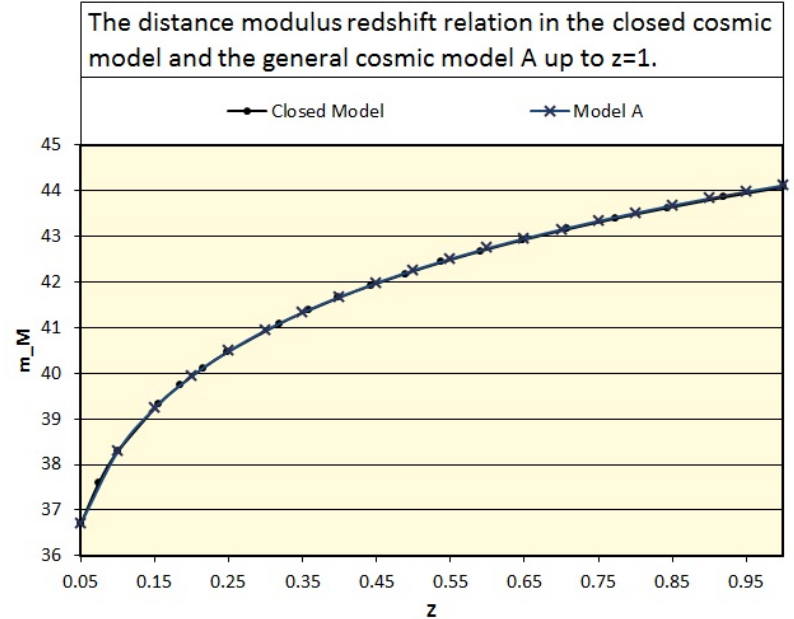

Figure 4. The distance modulus redshift relation in the closed cosmic model and the general cosmic model $A$ up to $z=$ 1.

1) The horizon distance of the universe $d_{h}\left(t_{o}\right)=14.4420_{-0.6231}^{+0.4916} \mathrm{Gpc}$.

2) The total mass within $d_{h}\left(t_{0}\right)$ is $M_{h}\left(t_{o}\right)=352.0373_{-29.4804}^{+34.0071} \times 10^{22} \mathrm{M}_{\odot}$.

3) The mass of matter in within $d_{h}\left(t_{0}\right)$ is $M_{m}\left(t_{o}\right)=112.5813_{-16.3806}^{+17.3288} \times 10^{22} \mathrm{M}_{\odot}$.

4) Number of the Milky Way-like galaxies equivalent to the mass of matter within $d_{h}\left(t_{0}\right)$ is

$N_{M W}=1407.2666_{-204.7570}^{+216.605} \times 10^{9}$.

5) Number of the Coma-like cluster of galaxies equivalent to the mass of matter within $d_{h}\left(t_{0}\right)$ is $N_{\text {COMA }}=562.9067_{-81.9028}^{+86.6438} \times 10^{6}$.

Errors in these estimations were obtained by similar way as in [1].

\section{Conclusion}

In this paper distributions of the proper distance, luminosity distance, angular diameter distance and distance modulus were investigated in terms of the redshift of an extragalactic object in the light of the closed cosmic model which was constructed in a previous paper. It is found that all of these cosmological distances increase continuously with redshift except the angular diameter distance which steeply increases with redshift towards maximum value $d_{A_{\max }}=1727.3954 \mathrm{Mpc}$ at $z_{\max }=1.6083$, then it decreases gradually with increasing $z$. It is also found that the luminosity distance of the extragalactic object in the closed cosmic model, observed model and $\Lambda=0$ model are approximately similar up to $z=0.1535$. However, the luminosity distance in the closed cosmic model approximately agrees with its value in the observed mo- 
del up to $z=0.6442$. Evaluations of the horizon distance of the universe, the total mass and the mass of matter within the horizon distance, the equivalent numbers of the Milky Way-like galaxies and the Coma-like clusters of galaxies to the mass of matter were calculated in the closed cosmic model at the present time.

\section{Acknowledgements}

This paper was funded by the Deanship of Scientific Research (DSR), King Abdulaziz University, Jeddah. The author, therefore, acknowledges with thanks DSR tech- nical and financial support.

\section{REFERENCES}

[1] F. A. Bukhari, "Cosmological Distances in Five General Cosmic Models," International Journal of Astronomy and Astrophysics, 2013.

[2] F. A. Bukhari, "A Closed Model of the Universe," International Journal of Astronomy and Astrophysics, 2013.

[3] F. A. Bukhari, "Five General Cosmic Models," Journal of King Abdulaziz University: Science, 2013. 